\title{
Evaluation of Age-Specific Efficacy and Safety of 12 Months of Sitagliptin Therapy Administered by Non-Diabetologists
}

\author{
Shouhei Yuasa ${ }^{a}$, Kazuyoshi Sato ${ }^{a}$, Takamoto Furuki ${ }^{a}$, Kosuke Minamizawa $^{a}$, Hiroyuki Sakai ${ }^{a}$, \\ Yuichi Numata ${ }^{\text {a }}$ Keiichi Chin ${ }^{\text {a }}$, Jisho Kojima ${ }^{\text {a }}$, Masaaki Miyakawa ${ }^{\text {a }}$, Ikuro Matsuba ${ }^{\text {b, c }}$
}

\begin{abstract}
Background: The mean age of overall incident dialysis patients is 68.9 years for men and 71.4 years for women, and it is increasing yearly, and diabetic nephropathy is the most common underlying disease of it. On the other hand, the number of diabetologist has been insufficient for an ever-increasing number of diabetic patients in Japan. In this context, the examination and treatment of diabetes in non-specialist settings, as well as in specialist settings, have become important.
\end{abstract}

Methods: A multicenter, retrospective, observational study was conducted in Kanagawa, Japan. Laboratory data were compiled for 1 year at intervals of 3 months following the start of sitagliptin treatment. The subjects were divided into three groups by age: $<65$ years $(\mathrm{n}=$ $148), 65-74$ years $(n=134)$, and $\geq 75$ years $(n=127)$. Laboratory values in comparison with baseline were compared within groups at various time points, and changes from baseline were compared among the different groups. Participating doctors were not diabetologists

Results: In the $65-74$ and $\geq 75$ years of age groups, estimated glomerular filtration rate (eGFR) was decreased significantly from baseline at months $3,6,9$, and 12 , whereas there was no significant decrease from the month-3 value at months 6,9 , or 12 . Meanwhile, in the $<65$ years of age group, eGFR was decreased significantly from baseline at months 9 and 12, whereas changes at months 3 and 6 were not significant. There were significant differences in the magnitude of eGFR changes among age groups at month 3, but not at months 6,9 , or 12 . Hemoglobin A1c (HbA1c) was reduced significantly at month 3 , and the reduced level was maintained until month 12 in all age groups.

Conclusions: The efficacy and safety of sitagliptin therapy in the elderly were confirmed in non-specialist settings. These findings will

Manuscript submitted January 23, 2019, accepted February 3, 2020

aStudy Group of the Hypertension Committee, Kanagawa Physicians Association, Kanagawa, Japan

bStudy Group of the Diabetes Committee, Kanagawa Physicians Association, Kanagawa, Japan

${ }^{\mathrm{c} C o r r e s p o n d i n g ~ A u t h o r: ~ I k u r o ~ M a t s u b a, ~ K a n a g a w a ~ D i a b e t e s ~ T a s k ~ F o r c e, ~}$ Kanagawa Physicians Association, 1-3 Fujimichou, Nakaku, Yokohama, Kanagawa 231-0037, Japan. Email: ikuro@matsuba-web.com

doi: https://doi.org/10.14740/jem634 help non-diabetologists choose an appropriate drug in areas where the numbers of diabetic patients and elderly persons are increasing.

Keywords: Type 2 diabetes mellitus; DPP-4 inhibitor; Non-specialist; Elderly; Diabetic nephropathy

\section{Introduction}

The number of diabetic patients in Japan was 2,371,000 in 2008 [1], and reached the highest ever in 2017 at 3,289,000 (1,848,000 men and 1,442,000 women) [2], an increase of approximately $39 \%$ over the 9 -year period, or approximately 102,000 more patients per year. The Japan Diabetes Society had a membership of 15,747 physicians as of 2019 [3], including 5,967 diabetologists [4]. The number of specialists has been insufficient for an ever-increasing number of diabetic patients. Furthermore, in rural areas, which already suffer from physician scarcity, patients who live far from a center that provides specialist care may present to non-diabetologists. In this context, the examination and treatment of diabetes in non-specialist settings, as well as in specialist settings, have become important.

Among the various complications associated with the progression of diabetes, diabetic nephropathy and its progression to dialysis have been particularly problematic in recent years. Diabetic nephropathy is the most common underlying disease in incident dialysis patients $(45.6 \%$ for men and $35.8 \%$ for women) [5]. The mean age of overall incident dialysis patients is 68.9 years for men and 71.4 years for women, and it is increasing yearly. When incident dialysis patients are stratified into 5-year age groups, the highest proportion of patients is found in the 75 - 79 years of age group for men and in the 80 84 years of age group for women. The effects of pharmacological therapy and the drug-induced renal damage should be kept in mind [6], and diabetes treatment in consideration of effects on the kidney is a critical challenge in elderly patients.

In Japan, the mainstay of diabetes treatment is dipeptidyl peptidase-4 (DPP-4) inhibitors. DPP-4 inhibitors increase blood incretin levels by selectively inhibiting DPP-4, an enzyme that inactivates incretin in vivo, and thereby stimulate glucose-dependent insulin secretion [7]. Sitagliptin was approved as the first DPP-4 inhibitor in Japan in 2009. Sitagliptin demonstrates durable efficacy with once-daily administra- 
tion, and is not likely to cause hypoglycemia or body weight (BW) change [8]. These characteristics make glycemic control easier for both physicians and patients, and the agent has become widely used as it matches needs of both diabetologists and non-diabetologists.

We accumulated data on 12-month treatment of diabetic patients with sitagliptin in non-diabetologist settings in the A Study of Safety and Efficacy of Sitagliptin Added to Insulin Therapy in the Treatment of Type 2 Diabetes in Kanagawa (ATTEST-K) study. After comparing three observational studies, including ATTEST-K, we reported that despite differences in patient characteristics, diabetes treatment type (with/without insulin), and the level of specialist care (diabetologist/non-diabetologist) among the studies, consistent reduction in hemoglobin A1c (HbA1c) by sitagliptin was confirmed [9].

However, age-specific analysis of the efficacy of sitagliptin treatment in non-specialist settings has not been reported. Thus, in the present study, we analyzed data from the ATTEST$\mathrm{K}$ study according to age groups to evaluate the efficacy in glycemic control and the safety, including effects on the kidney of sitagliptin in elderly patients in non-specialist settings.

\section{Materials and Methods}

\section{Study design}

A multicenter, retrospective, observational study was conducted at clinics and hospitals that were members of the Kanagawa Physicians Association. Data were collected from the medical records of the subjects, and the follow-up period was 1 year after oral informed consent was obtained. This study was approved by the Ethics Review Board of the Kanagawa Physicians Association. This study was undertaken in accordance with the Ethical Guidelines for Clinical Studies of the Japanese Ministry of Health, Labour and Welfare as well as with the Helsinki Declaration. The study period was from September 30, 2012 to October 31, 2013, and participating doctors were not diabetes specialists.

\section{Patients}

Patients were eligible for this study if they had type 2 diabetes mellitus (T2DM), were aged 20 years or older, regularly attended a clinic or hospital with membership in the Kanagawa Physicians Association, and received treatment with sitagliptin for 1 year or longer. Sitagliptin was started if glycemic control was inadequate for at least 1 month despite diet and exercise therapy or diet and exercise plus oral antidiabetic drugs. The exclusion criteria were as follows: a history of hypersensitivity to any component of sitagliptin; a history of severe ketoacidosis or diabetic coma or precoma within 6 months before the start of sitagliptin therapy; severe infection; recent or planned surgery or severe trauma; concurrent use of glinides; and patients whom the attending doctor considered to be inappropriate for this study for other reasons.

\section{Items investigated}

The baseline characteristics investigated for the subjects included gender, age, duration of diabetes, complications, smoking history and alcohol history. Use of sitagliptin and other antidiabetic drugs was assessed before treatment with sitagliptin, at the start of treatment, and 12 months after the start of treatment. Efficacy endpoints were determined at each of the specified times, including HbA1c (National Glycohemoglobin Standardization Program value), blood glucose (fasting and postprandial), serum lipids (total cholesterol (TC), low-density lipoprotein cholesterol (LDL-C), high-density lipoprotein cholesterol (HDL-C), and triglycerides (TGs)), BW, office systolic blood pressure (SBP), office diastolic blood pressure (DBP), office pulse rate (PR), morning home SBP, morning home DBP, morning home PR, evening home SBP, evening home $\mathrm{DBP}$, and evening home PR. In addition, serum creatinine, estimated glomerular filtration rate (eGFR), urine albumin-tocreatinine ratio and adverse events were evaluated at each of these times to assess safety.

\section{Statistical analysis}

After exclusion of patients for whom age data were not available from the collected case records, the remaining patients formed the safety analysis set. The efficacy analysis set was obtained by excluding patients who met either of the following criteria from the safety analysis set: 1) No HbAlc data at the start of sitagliptin treatment; or 2) No HbA1c data at month 12.

Data were statistically analyzed in three age groups: $<65$ years, $65-74$ years, and $\geq 75$ years.

Patient characteristics were compared using analysis of variance (ANOVA) for continuous variables and Chi-squared test for nominal variables. With regard to the efficacy in each of the three groups, a paired $t$-test was used to evaluate the changes in various parameters from baseline at months 3,6 , 9 , and 12, and ANOVA was used for intergroup comparisons. With regard to eGFR, changes from month-3 values at months 6,9 , and 12 after treatment initiation were also evaluated by paired $t$-test. Intra-group comparisons did not take multiplicity into account, and the significance level was set at 5\% (twosided). The analytical software R, version 3.4.1 (R Foundation for Statistical Computing, Vienna, Austria (https://www.Rproject.org/)), was used for calculations. Data are presented as mean \pm standard deviation or number (proportion) of patients.

\section{Results}

A total of 530 patients were enrolled and 121 patients were excluded. The remaining 409 patients formed the efficacy analysis set (Supplementary Material 1, www.jofem.org). Baseline characteristics of the subjects are shown in Table 1. Study population breakdown by age group was as follows: 148 patients $(36.2 \%)$ at $<65$ years, 134 patients $(32.8 \%)$ at $65-74$ years, and 127 patients at $\geq 75$ years $(31.0 \%)$. The proportion of patients with hypertension or cerebrovascular disorders and the 


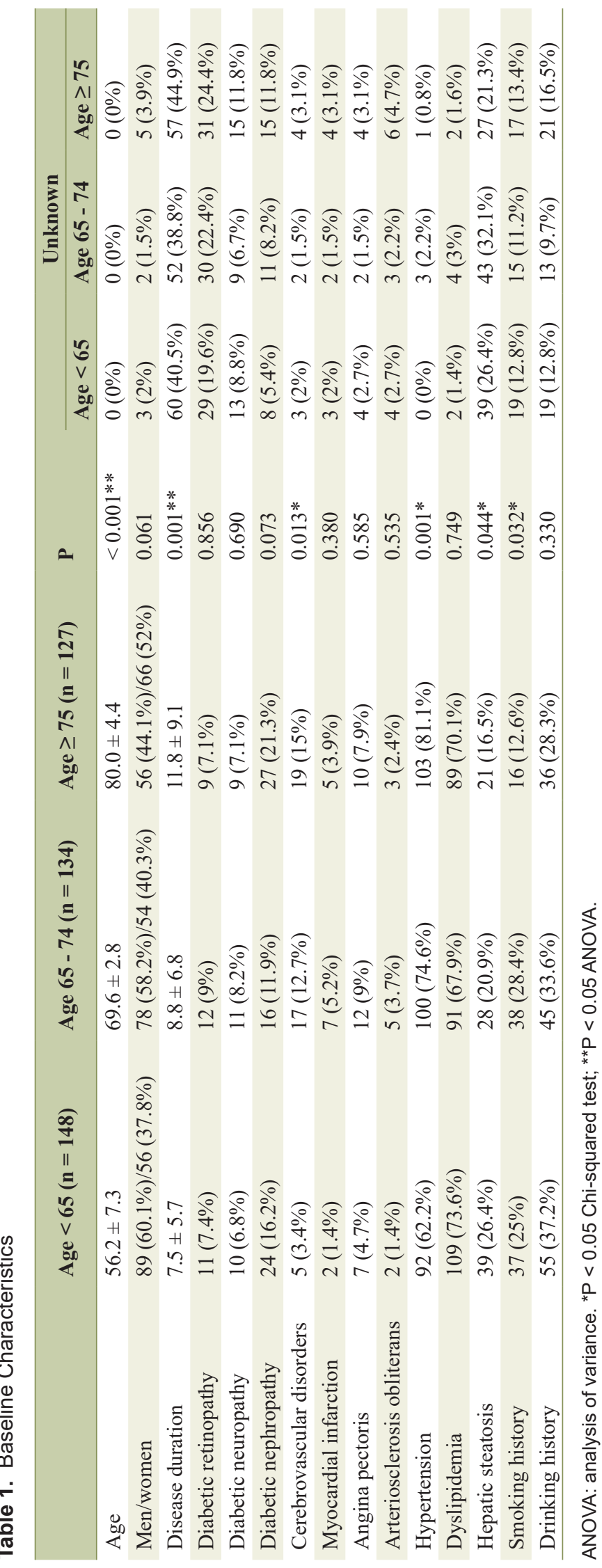

disease duration increased with increasing age, and the proportion of patients with hepatic steatosis was higher at younger age. Smoking history was lower at $\geq 75$ years.

Medication data from before to 12 months after the start of the treatment are shown in Table 2. The dose of sitagliptin at the start of the study tended to be lower with increasing age; however, there was no significant difference among age groups. Medications that were discontinued at the start of sitagliptin therapy were mostly thiazolidines, $\alpha$-glucosidase inhibitors ( $\alpha$-GIs), and glinides. Changes of concomitant medications made in five or more patients after the start of the study were the addition of biguanides in the $<65$ and $65-74$ years of age groups, and the addition of sulfonylureas in the $\geq 75$ years of age group.

Changes in eGFR according to age group are shown in Figure 1. In the $65-74$ and $\geq 75$ years of age groups, eGFR was decreased significantly from baseline at months $3,6,9$, and 12 , whereas there was no significant decrease from the month-3 value at months 6,9 , or 12 . Meanwhile, in the $<65$ years of age group, eGFR was decreased significantly from baseline at months 9 and 12, whereas changes at months 3 and 6 were not significant. There were significant differences in the magnitude of eGFR changes among age groups at month 3 , but not at months 6,9 , or 12 (Fig. 2).

Changes in other laboratory parameters are shown in Supplementary Material 2 (www.jofem.org). HbAlc was reduced significantly at month 3 , and the reduced level was maintained until month 12 in all age groups. There were significant differences in the magnitude of $\mathrm{HbA} 1 \mathrm{c}$ changes at month 9 only, and not at other time points. The fasting and postprandial blood glucose levels improved significantly in each age group.

The BW was decreased significantly at months 6 and 12 in the $\geq 75$ years of age group, whereas changes at other time points in this group and changes in other age groups were not significant. As for other laboratory parameters, significant changes observed consistently within the groups were significant increase in serum creatinine levels in the $65-74$ and $\geq 75$ years of age groups and significant decrease in LDL-C levels in the $65-74$ years of age group. While significant differences were observed among age groups in the magnitude of changes in fasting blood glucose at month 3 and serum creatinine at month 9, no difference was observed among groups in the magnitude of changes in other parameters.

There were significant decreases in some of the home SBP and DBP values measured in morning and evening, whereas changes in home PR measured in morning and evening were not significant (Supplementary Material 3, www.jofem.org).

As for adverse events for which causality cannot be ruled out, hypoglycemia was reported in two patients in the $<65$ years of age group and in one patient in the $65-74$ years of age group. Constipation was reported in two, two, and three patients in the $<65,65-74$, and $\geq 75$ years of age groups, respectively. One patient each in the three groups discontinued treatment due to the adverse event (constipation).

\section{Discussion}

The present study demonstrated the efficacy and safety of sit- 
Table 2. Medications in the Efficacy Analysis Set

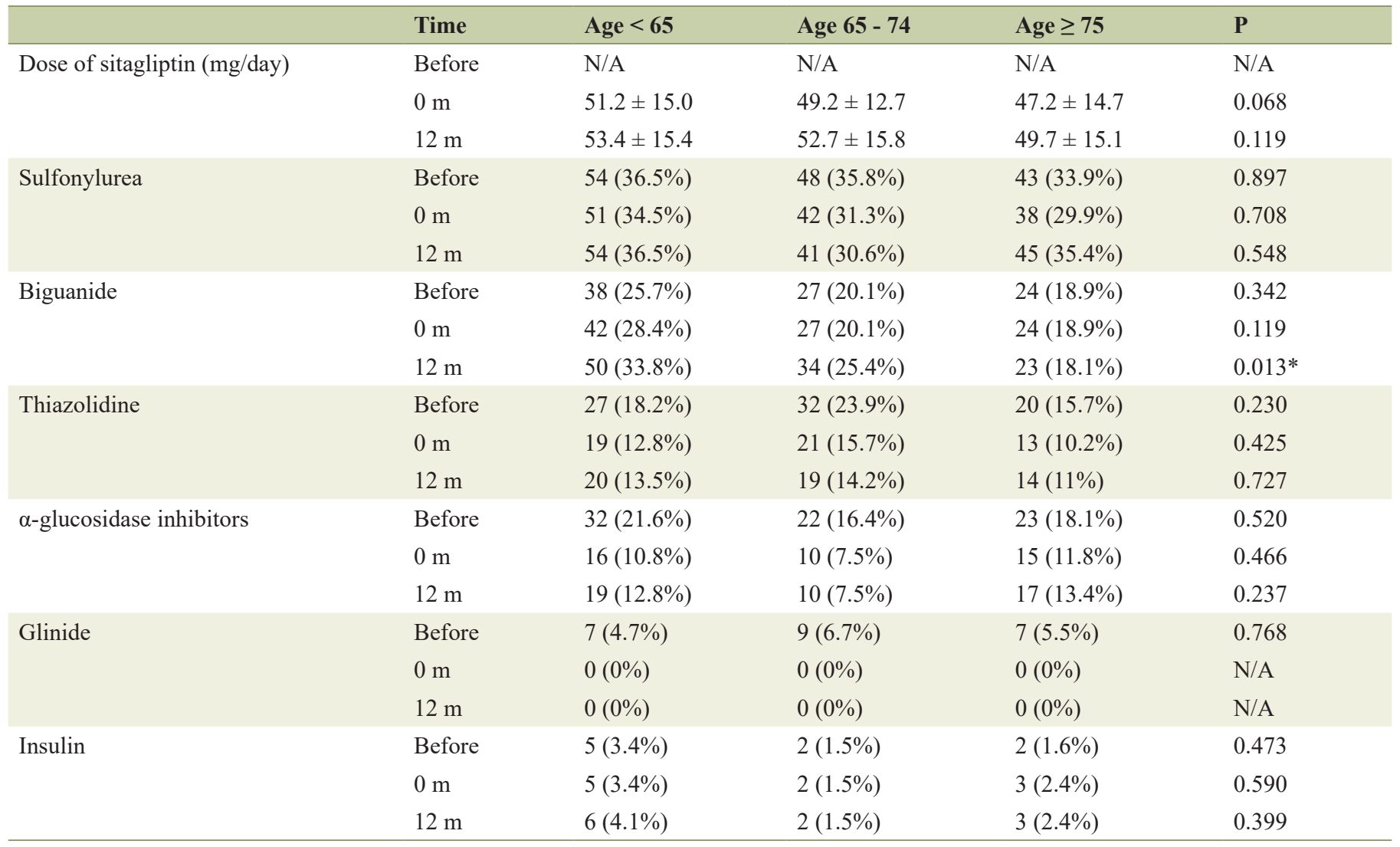

N/A: not available; m: months. ${ }^{*} \mathrm{P}<0.05$ Chi-squared test.

agliptin treatment in Japanese elderly patients administered by non-diabetologists.

As for intra-group changes in eGFR, significant decrease was observed in the $65-74$ and $\geq 75$ years of age groups at month 3, and after this time point, the level remained flat without additional decreases. Meanwhile, in the $<65$ years of age group, eGFR was significantly decreased first at month 9 and then remained flat until month 12 . Also in the study of sitaglip-

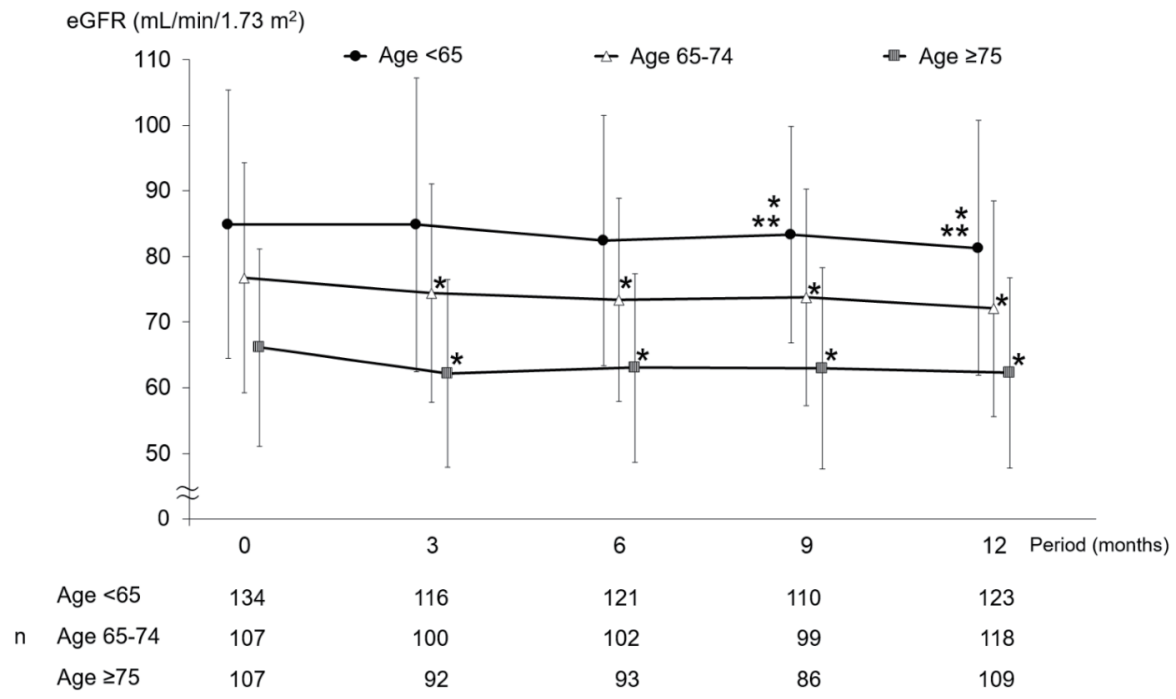

Figure 1. Changes in eGFR. ${ }^{*} \mathrm{P}<0.05$ versus month 0 paired $t$-test; ${ }^{* *} \mathrm{P}<0.05$ versus month 3 paired $t$-test. eGFR: estimated glomerular filtration rate. 


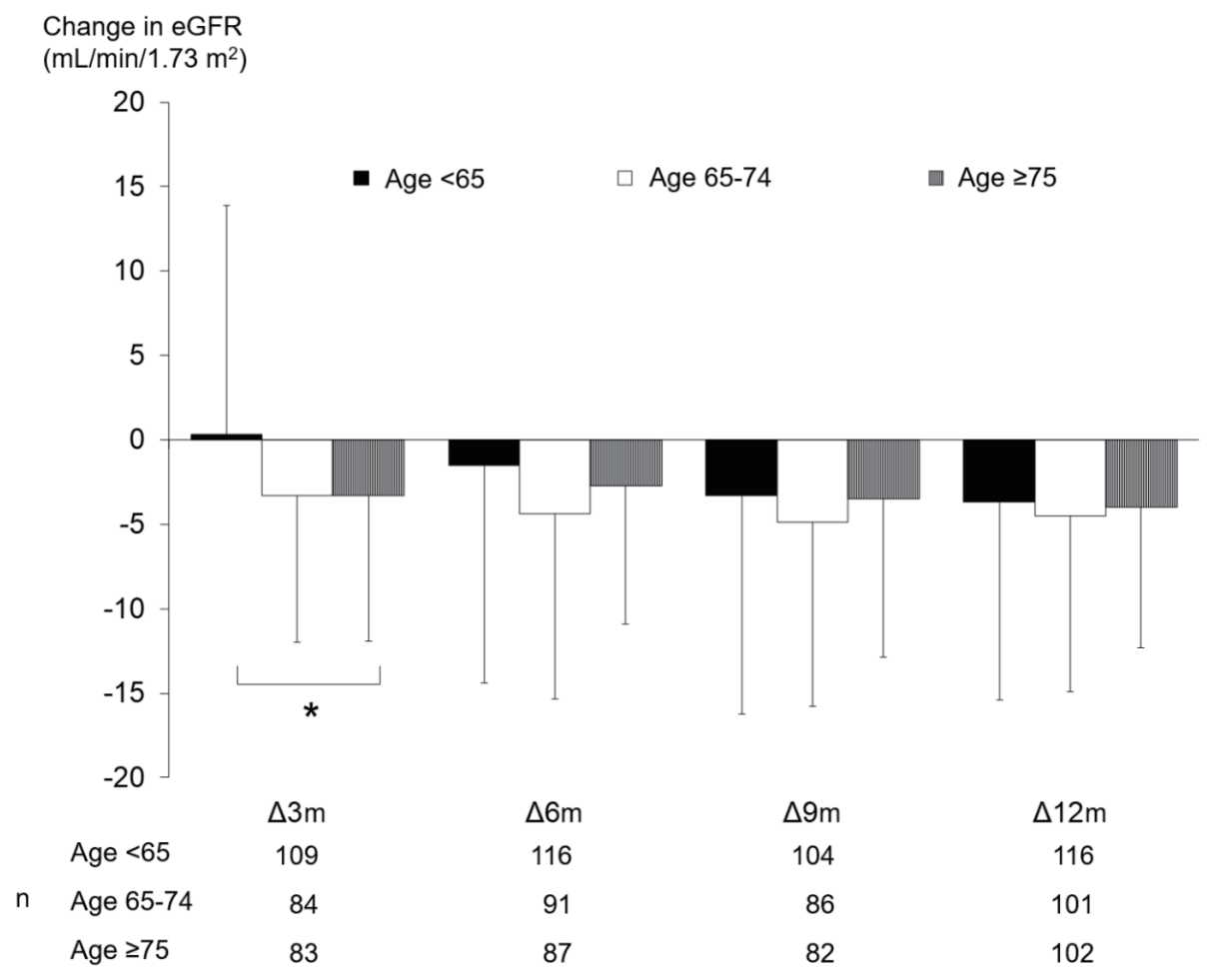

Figure 2. Amounts of change in eGFR. ${ }^{*} \mathrm{P}<0.05$ ANOVA. eGFR: estimated glomerular filtration rate; ANOVA: analysis of variance; m: months.

tin treatment by diabetologists (ASSET-K), eGFR decreased significantly and serum creatinine increased significantly in each of the $<65,65-74$, and $\geq 75$ years of age groups [10]. In that report, detailed changes in creatinine were described: the level was increased at month 3, slightly decreased from months 3 to 6 , and thereafter remained flat in the $65-74$ years and $\geq 75$ years of age groups. Meanwhile, in the $<65$ years of age group, the level showed a very gradual increasing trend without decline from months 3 to 6 . Considering the inverse relationship between eGFR and serum creatinine, the findings described above are closely similar to the results of the present study.

We previously reported that the level of specialist care (diabetologists or non-diabetologists) did not affect the eGFR changes associated with sitagliptin [9], and it seems that a similar finding was obtained in our age-specific analysis of eGFR. Although the mechanism underlying the difference in the timing of changes among age groups is unclear, the onset of effects on the renal function may be earlier in the elderly. Nevertheless, the level remains flat thereafter and thus the drug is safe for use. In the present study, urinary albumin was decreased significantly at month 9 in the $<65$ years of age group, whereas there was no significant change in the other two groups, indicating that effects on the kidney are limited. Besides the 1-year follow-up in the present study, the long-term analysis of the ASSET-K study described above showed no significant change in creatinine between 6 months and 2 years after the start of treatment [11]. Results from the Trial Evaluating Cardiovascular Outcomes with Sitagliptin (TECOS) study showed that change in eGFR was $-4.0 \pm 18.4 \mathrm{~mL} / \mathrm{min} / 1.73 \mathrm{~m}^{2}$ at 4 years after the start of treatment [12], also indicating that long-term therapy with sitagliptin can be considered not to induce excessive decrease in the glomerular filtration rate.

While the renal safety was not compromised in non-specialist settings in the present study, some reports noted provision of more appropriate treatment by diabetologists. A survey on the diabetic care by diabetologists and non-diabetologists in Chiba, Japan reported that among patients indicated for albuminuria test for diagnosis of early nephropathy, $10 \%$ of the patients managed by diabetologists and $37 \%$ of those managed by non-diabetologists did not undergo the test [13]. It is important to remember that the optimal treatment can only be achieved by providing an appropriate test and drawing on clinical data. In an evaluation of non-diabetologists who participated in a 15-month education program, increased provision of albuminuria screening by $29.8 \%$, as well as the improvement in glycemic control status, were reported [14]. Non-specialists may raise the level of their diabetic care by participating in such education programs.

$\mathrm{HbAlc}$ was significantly reduced in all age groups in the non-specialist settings of the present study. Significant reductions in $\mathrm{HbAlc}$ in all age groups were also reported in the studies of sitagliptin treatment by diabetologists $[10,15]$. However, the magnitude of changes at month 9 was different among age groups in the present study. HbAlc showed a greater decrease in patients with a higher baseline $\mathrm{HbAlc}$, shorter duration of disease and older age in our previous report, and the most influential factor was baseline HbA1c [9]. In the pre- 
sent stratified analysis, baseline HbA1c was higher in the $<65$ years of age group compared with the other two groups and may have affected the results. Furthermore, in this age-specific analysis, HbA1c was less than $7 \%$ at month 9 in the $65-74$ and $\geq 75$ years of age groups, indicating that glycemic control was achieved. DPP-4 inhibitors enhance insulin secretion in a glucose level-dependent manner through the inhibition of GLP-1 inactivation and the DPP-4-mediated effect, and inhibit glucagon secretion to improve glycemic control [16, 17]. This characteristic glucose-dependent action is reflected in the findings of the present study.

In the present study, hypoglycemia occurred only in two patients in the $<65$ years of age group and one in the $65-74$ years of age group. While elderly patients on hypoglycemic agents sometimes have trouble with hypoglycemia caused by a delay in drug excretion due to reduced renal function, the findings of the present study demonstrated the safety of sitagliptin treatment in the elderly in non-specialist settings. Other side effects included seven cases of constipation, three of which led to treatment discontinuation; there was no difference among age groups. A previous report on specialists also found no difference in rates of side effects among age groups [15]. However, in patients aged $\geq 75$ years, cognitive or physical function is often impaired [18-22], and symptoms may be overlooked. We should encourage patients to be aware of subjective symptoms of side effects, so as not to miss their signs.

In the $\geq 75$ years of age group, BW was decreased significantly at month $6(-0.55 \pm 2.23 \mathrm{~kg})$ and at month $12(-0.57 \pm$ $2.14 \mathrm{~kg})$. However, significant change at month 9 or continuous decline was not observed, and the magnitude of reductions remained at around $0.5 \mathrm{~kg}$; these changes are not considered to reflect effects on the safety. The finding that there was no difference in the magnitude of weight changes among groups is similar to the results reported previously in the studies of specialists $[10,15]$.

LDL-C decreased significantly in the 65 - 74 years of age group. While significant changes at all measurement time points were observed only in this group, decreasing trend was observed in the $<65$ and $\geq 75$ years of age groups and no difference in the magnitude of changes was observed among groups, indicating that the $65-74$ years of age group may not be special. A previous report described the decrease in LDL$\mathrm{C}$ in patients aged $\geq 65$ years [23], and a similar change was found in non-specialist settings in the present study.

As for office BP, decreasing trend in each group and significant decreases at some time points were observed, and the magnitude of changes did not differ among groups. An overall analysis without stratification by age reported significant decreases in office SBP at months 6 and 12 and in office DBP at months 3, 6, 9, and 12 [24]. Decreased number of time points at which significant changes were observed after the stratification might be attributable to the division of patients.

\section{Limitations}

The present study lacked a control group, because it was conducted in an open-label, retrospective fashion. In addition, no data on concomitant use with a glinide and a sodium-glucose cotransporter-2 (SGLT2) inhibitor were available. Glinides were not approved for use with sitagliptin at the start of the study. SGLT2 inhibitors were also not approved at the start of the study. The study was not based on accurate power calculations because of its nature as an exploratory analysis.

\section{Conclusions}

The efficacy and safety of sitagliptin therapy in the elderly were confirmed in non-specialist settings. These findings will help non-diabetologists choose an appropriate drug in areas where the numbers of diabetic patients and elderly persons are increasing.

\section{Supplementary Material}

Suppl 1. Participant Flow.

Suppl 2. Efficacy Endpoints.

Suppl 3. Change in Home SBP, DBP, PR.

\section{Acknowledgments}

We would also like to thank all the ATTEST-K study group investigators who contributed to this research, as well as the Hypertension and Renal Disease Committee Study Group of the Kanagawa Physicians Association.

\section{Financial Disclosure}

This research was supported by funds from the Japan Kidney Foundation and a grant from the Japan Renal Foundation.

\section{Conflict of Interest}

The authors declare no conflict of interest.

\section{Informed Consent}

Oral informed consent was obtained from the patients.

\section{Author Contributions}

MM and IM conceived and designed the experiments; SY, KS, TF, KM, HS, YN, KC, JK, and MM enrolled the patients; IM analyzed the data; SY wrote the first draft of the manuscript; SY, MM, and IM contributed to the writing of the manuscript; SY, KS, TF, KM, HS, YN, KC, JK, MM, and IM agree with the manuscript's results and conclusions. All authors have read and confirmed that they meet the ICMJE criteria for authorship. 


\section{Data Availability}

The biochemical data used to support the findings of this study are available from the corresponding author upon request.

\section{References}

1. Ministry of Health, Labour and Welfare. Overview of patient survey in 2008. Retrived from: https://www.mhlw. go.jp/toukei/saikin/hw/kanja/08/dl/05.pdf Accessed 8 January 2020.

2. Ministry of Health, Labour and Welfare. Overview of patient survey in 2017. Retrived from: https://www.mhlw. go.jp/toukei/saikin/hw/kanja/17/dl/05.pdf Accessed 8 January 2020.

3. The Japanese Assosiation of Medical Science. Japan Medical Association Subcommittee Information. Retrived from: http://jams.med.or.jp/members-s/51.html Accessed 8 January 2020.

4. The Japan Diabetes Society. Specialist search. Retrived from: http://www.jds.or.jp/modules/senmoni/ Accessed 8 January 2020.

5. The Japanese Society for Dialysis Therapy. Current status of dialysis therapy. 2017. Retrived from: https://docs.jsdt. or.jp/overview/ Accessed 8 January 2020.

6. Choudhury D, Ahmed Z. Drug-associated renal dysfunction and injury. Nat Clin Pract Nephrol. 2006;2(2):80-91.

7. Scheen AJ. A review of gliptins for 2014. Expert Opin Pharmacother. 2015;16(1):43-62.

8. Scheen AJ. Safety of dipeptidyl peptidase-4 inhibitors for treating type 2 diabetes. Expert Opin Drug Saf. 2015;14(4):505-524.

9. Yuasa S, Sato K, Takai M, Ishikawa M, Umezawa S, Kubota A, Maeda H, et al. Factor Analysis of Changes in Hemoglobin A1c After 12 Months of Sitagliptin Therapy in Patients With Type 2 Diabetes. J Clin Med Res. 2016;8(6):461-471.

10. Umezawa S, Kubota A, Maeda H, Kanamori A, Matoba $\mathrm{K}$, Jin Y, Minagawa F, et al. Two-year assessment of the efficacy and safety of sitagliptin in elderly patients with type 2 diabetes: Post hoc analysis of the ASSET-K study. BMC Endocr Disord. 2015;15:34.

11. Maeda H, Kubota A, Kanamori A, Tanaka Y, Terauchi Y, Matsuba I, Study Group of Diabetes Committee KPA. Effects of sitagliptin on the serum creatinine in Japanese type 2 diabetes. Diabetes Res Clin Pract. 2015;108(3):e42-45.

12. Green JB, Bethel MA, Armstrong PW, Buse JB, Engel SS, Garg J, Josse R, et al. Effect of Sitagliptin on Cardiovascular Outcomes in Type 2 Diabetes. N Engl J Med. 2015;373(3):232-242.

13. Kanatsuka A, Mimura M, Sinomiya M, Hashimoto N, Kuribayashi N, Sakurai K, Suzuki H, et al. Survey of diabetic clinics in Chiba prefecture performed by dia- betologists and general physicians. J. Japan Diab. Soc. 2012;55(9):671-680.

14. Ching D, Forte D, Aitchison E, Earle K. Are there longterm benefits of experiential, interprofessional education for non-specialists on clinical behaviours and outcomes in diabetes care? A cohort study. BMJ Open. 2016;6(1):e009083.

15. Takai M, Ishikawa M, Maeda H, Kanamori A, Kubota A, Amemiya $\mathrm{H}$, Iizuka T, et al. Efficacy and safety of adding sitagliptin in type 2 diabetes patients on insulin: agestratified comparison at one year in the ASSIST-K study. J Clin Med Res. 2019;11(5):311-320.

16. Drucker DJ. Enhancing incretin action for the treatment of type 2 diabetes. Diabetes Care. 2003;26(10):29292940.

17. Herman GA, Bergman A, Stevens C, Kotey P, Yi B, Zhao $\mathrm{P}$, Dietrich B, et al. Effect of single oral doses of sitagliptin, a dipeptidyl peptidase-4 inhibitor, on incretin and plasma glucose levels after an oral glucose tolerance test in patients with type 2 diabetes. J Clin Endocrinol Metab. 2006;91(11):4612-4619.

18. Araki A. Low well-being, cognitive impairment and visual impairment associated with functional disabilities in elderly Japanese patients with diabetes mellitus. Geriatrics \& Gerontology International. 2004;4(1):15-24.

19. Ma F, Wu T, Miao R, Xiao YY, Zhang W, Huang G. Conversion of mild cognitive impairment to dementia among subjects with diabetes: a population-based study of incidence and risk factors with five years of follow-up. J Alzheimers Dis. 2015;43(4):1441-1449.

20. Solini A, Penno G, Bonora E, Fondelli C, Orsi E, Trevisan R, Vedovato M, et al. Age, renal dysfunction, cardiovascular disease, and antihyperglycemic treatment in type 2 diabetes mellitus: findings from the Renal Insufficiency and Cardiovascular Events Italian Multicenter Study. J Am Geriatr Soc. 2013;61(8):1253-1261.

21. Huang ES, Liu JY, Moffet HH, John PM, Karter AJ. Glycemic control, complications, and death in older diabetic patients: the diabetes and aging study. Diabetes Care. 2011;34(6):1329-1336.

22. Lind M, Olsson M, Rosengren A, Svensson AM, Bounias I, Gudbjornsdottir S. The relationship between glycaemic control and heart failure in 83,021 patients with type 2 diabetes. Diabetologia. 2012;55(11):2946-2953.

23. Ujihara N, Sakura H, Hashimoto N, Sasamoto K, Ohashi $\mathrm{H}$, Hasumi S, Kasahara T, et al. Erratum to: Efficacy and safety of sitagliptin in elderly patients with type 2 diabetes mellitus and comparison of hypoglycemic action of concomitant medications: a subanalysis of the JAMP study. Diabetol Int. 2018;9(1):66-67.

24. Yuasa S, Sato K, Furuki T, Minamizawa K, Sakai H, Numata Y, Chin K, et al. Primary care-based investigation of the effect of sitagliptin on blood pressure in hypertensive patients with type 2 diabetes. J Clin Med Res. 2017;9(3):188-192. 